\title{
El desarrollo humano y los movimientos migratorios en las culturas mediterráneas
}

\author{
María Teresa Algado Ferrer \\ Raúl Ruiz Callado \\ Universidad de Alicante. Departamento de Sociología I \\ MT.Algado@ua.es \\ RaulRuiz@ua.es
}

Recibido: 17-04-2007

Aceptado: 25-06-2008

\section{Resumen}

Los autores de este trabajo toman como marco de estudio e interacción el espacio físico, social y cultural mediterráneo para, en primer lugar, analizar descriptiva y comparativamente los logros obtenidos en educación, salud, recursos económicos e igualdad de género que indican su nivel de desarrollo socio-cultural y de bienestar humano, y que unen y separan a sus poblaciones. En segundo lugar, se verifica la existencia en los distintos países mediterráneos de profundos desequilibrios demográficos y de desiguales niveles de desarrollo y bienestar que son algunos de los factores que favorecen, aunque no de forma única, los movimientos migratorios.

Palabras clave: sociología de la cultura, desequilibrio demográfico, bienestar, desigualdades.

\section{Abstract. Human Development and Migration in Mediterranean Cultures}

This work aims, in the first place, to analyze, describe, and compare the achievements in education, public health, economic resources, and gender equality that have taken place in the Mediterranean space (physical, social, and cultural). These achievements indicate the level of socio-cultural development and human welfare of this space. In the second place, it shows that there are deep demographic imbalances and unequal levels of development and welfare between different Mediterranean countries. It is showed how these are some of the factors that partially favour migration.

Key words: sociology of culture, demographical imbalances, welfare, inequalities.

\section{Sumario}

Introducción: el Mediterráneo como objeto de estudio

1. El desarrollo humano en el Mediterráneo

2. El Mediterráneo: un mar de migraciones
3. Las remesas mediterráneas, oportunidad para evitar los desequilibrios sociales

4. Conclusiones

Bibliografía 


\section{Introducción: el Mediterráneo como objeto de estudio}

Mar de desigualdades, mar de oportunidades económicas, gran lago de los desequilibrios demográficos, mar que une y separa, mar que refleja las contradicciones de un presente complejo, espacio en el que todo se mezcla y se recompone, el Mediterráneo ha sido definido y analizado desde la perspectiva literaria y desde las ciencias sociales enfatizando, bien lo que de común posee, bien lo que le diferencia. En esa unidad original, en términos braudelianos, que forma el Mediterráneo confluyen tres continentes, Europa, Asia y África y nacen las tres religiones monoteístas: cristianismo, islamismo y judaísmo.

La relevancia de este espacio común, tanto en los aspectos económicos como en los políticos o culturales, y la necesidad de crear una zona euromediterránea de cooperación y solidaridad se plasmó hace ya más de una década en la Conferencia Euromediterránea celebrada en Barcelona en 1995. La intención de la Declaración de Barcelona, apoyada por los quince países entonces miembros de la Unión Europea y doce países del sur y este del Mediterráneo, era reducir las desigualdades entre el norte y el sur del Mediterráneo y conseguir una zona de prosperidad compartida, de libre comercio y de paz y estabilidad. Un lugar en el que fuera posible el intercambio cultural y la cooperación en todos los aspectos, no solo vertical, norte-sur, sino también horizontal, sur-sur. Desde entonces, la Unión Europea ha establecido acuerdos de asociación con los distintos países socios del Mediterráneo (el primero de ellos fue firmado con Túnez en 1995) y ha puesto en marcha distintos programas de apoyo a los mismos para reducir las diferencias de riqueza (MEDA, EuropeAid, Iniciativa Eumedis, entre otros), que no han dado los frutos deseados sino que, por el contrario, han generado una situación de desconfianza y decepción en ambos lados del Mediterráneo. Pese a esto, los estudiosos y evaluadores del Proceso de Barcelona lo consideran un gran avance si se tienen en cuenta otras iniciativas políticas que le antecedieron.

La baja inversión extranjera directa que han recibido de la Unión Europea los socios mediterráneos del sur, al haberse ésta orientado más hacia los nuevos estados miembros del este de Europa, junto con la escasa colaboración de los países socios mediterráneos debido a sus rígidas estructuras políticas, económicas y sociales y a sus tímidas reformas económicas, han contribuido a reforzar las diferencias de riqueza entre las dos orillas; más aún si se tienen en cuenta los diferentes rasgos demográficos que caracterizan a ambas.

Tomando, pues, como marco de estudio e interacción ese espacio físico, social y cultural que denominamos el Mediterráneo, en este artículo se analizan los distintos logros conseguidos por los países que lo conforman en cuanto a la salud, la educación, los recursos económicos y la igualdad de género que, en definitiva, indican su nivel de desarrollo y de bienestar humano. Se parte de la idea de que muchos de los desequilibrios demográficos, -en el norte, países que, en su mayoría, han acabado su transición demográfica, tienen unos niveles de crecimiento, fecundidad y mortalidad bajos y unas estructuras de población envejecidas; en el sur, países transicionales, aunque con alguna excep- 
ción, con niveles de crecimiento, fecundidad y mortalidad todavía altos y con estructuras de población jóvenes-, y gran parte de los desiguales niveles de desarrollo y bienestar conseguidos en los distintos países son, en gran medida, si no los únicos factores que provocan los movimientos migratorios, sí al menos, algunos de los que los favorecen. En segundo lugar, se lleva a cabo un análisis de los flujos migratorios en el Mediterráneo, incidiendo especialmente en sus efectos socioeconómicos y profundizando en el papel que las remesas desempeñan, destacando sus beneficios y riesgos potenciales.

\section{El desarrollo humano en el Mediterráneo}

En este trabajo se utilizan dos de los indicadores propuestos por Naciones Unidas para medir el desarrollo humano: el Índice de Desarrollo Humano (IDH) y el Índice de Desarrollo relativo al Género (IDG) y se aplican a los países de las riberas norte, sur y este del Mediterráneo con fines comparativos. La limitación de estos índices es la imposibilidad de realizar comparaciones intertemporales al utilizar en la elaboración de los indicadores de cada año distintos criterios metodológicos, lo que obliga a realizar únicamente las comparaciones entre países en el mismo año. Los dos únicos países del Mediterráneo sobre los que no existe información de su IDH son Serbia y Montenegro.

En la Tabla 1 se presenta el Índice de Desarrollo Humano de los países del Mediterráneo con sus componentes y sus mediciones. Se ha conservado, al lado de cada país, el número que les identifica con la posición ocupada en el ranking mundial. Así pues, Francia, el país con mayor desarrollo humano del conjunto del Mediterráneo, ocupa por tanto la primera posición, pero la diez en el total mundial; Marruecos, ocupa la última posición al ser el país del Mediterráneo con menor desarrollo humano, y la ciento veintiséis respecto al total mundial.

Siguiendo los baremos acordados por Naciones Unidas, sólo catorce países de los que forman el Mediterráneo pueden considerarse de desarrollo humano alto (IDH superior a 0,800$)$ y nueve de desarrollo humano medio (IDH entre 0,500 y 0799). Ningún país del Mediterráneo, según estos criterios, pertenece al grupo de países de desarrollo humano bajo (IDH por debajo de $0,500)$.

De los catorce países que por su IDH se consideran de desarrollo humano alto, once están situados en la ribera norte y ocho de ellos pertenecen a la Unión Europea. La mayoría de los países de la ribera sur y este pertenecen al grupo de desarrollo humano medio.

Una línea de fractura claramente visible la constituye el desigual PIB per cápita de los países del norte y del sur del Mediterráneo. Así por ejemplo, Francia (país con el IDH más alto del Mediterráneo) tiene un PIB siete veces mayor que el de Marruecos (país con el IDH más bajo del Mediterráneo).

Los datos de la Tabla 1 permiten también comprobar que no siempre el ingreso nacional, medido a través del PIB, es el único o el mejor indicador para medir el bienestar humano, aunque exista entre ambos una estrecha rela- 
Tabla 1. Índice de Desarrollo Humano

\begin{tabular}{|c|c|c|c|c|c|c|c|c|}
\hline $\begin{array}{l}\text { Clasificación } \\
\text { según el IDH }\end{array}$ & $\begin{array}{l}\text { Valor del } \\
\text { IDH }\end{array}$ & $\begin{array}{l}\text { Esperanza de } \\
\text { vida al nacer } \\
\text { (años) }\end{array}$ & $\begin{array}{c}\text { Tasa de } \\
\text { alfabetización } \\
\text { (\% de } 15 \text { años y más })\end{array}$ & $\begin{array}{c}\text { Tasa bruta } \\
\text { combinada de } \\
\text { matriculación }\end{array}$ & $\begin{array}{l}\text { PIB per cápita } \\
\text { (PPA \$ USA) }\end{array}$ & $\begin{array}{c}\text { Índice de } \\
\text { esperanza } \\
\text { de vida }\end{array}$ & $\begin{array}{l}\text { Índice de } \\
\text { educación }\end{array}$ & $\begin{array}{l}\text { Índice } \\
\text { del PIB }\end{array}$ \\
\hline \multicolumn{9}{|l|}{ IDH alto } \\
\hline 10 Francia & 0,952 & 80,2 & - & 96,5 & 30.386 & 0,919 & 0,982 & 0,954 \\
\hline 13 España & 0,949 & 80,5 & - & 98 & 27.169 & 0,925 & 0,987 & 0,935 \\
\hline 20 Italia & 0,941 & 80,3 & 98,4 & 90,6 & 28.529 & 0,922 & 0,958 & 0,944 \\
\hline 23 Israel & 0,932 & 80,3 & 97,1 & 89,6 & 25.864 & 0,921 & 0,946 & 0,927 \\
\hline 24 Grecia & 0,926 & 78,9 & 96,0 & 99 & 23.381 & 0,898 & 0,97 & 0,91 \\
\hline 27 Eslovenia & 0,917 & 77,4 & 99,7 & 94,3 & 22.273 & 0,874 & 0,974 & 0,902 \\
\hline 28 Chipre & 0,903 & 79,0 & 96,8 & 77,6 & 22.699 & 0,900 & 0,904 & 0,905 \\
\hline 29 Portugal & 0,897 & 77,7 & 93,8 & 89,8 & 20.410 & 0,879 & 0,925 & 0,888 \\
\hline 34 Malta & 0,878 & 79,1 & 87,9 & 80,9 & 19.189 & 0,901 & 0,856 & 0,877 \\
\hline 47 Croacia & 0,850 & 75,3 & 98,1 & 73,5 & 13.042 & 0,839 & 0,899 & 0,813 \\
\hline 56 Libia & 0,818 & 73,4 & 84,2 & 94,1 & 10.335 & 0,806 & 0,875 & 0,774 \\
\hline 66. Bosnia-H. & 0,803 & 74,5 & 96,7 & 69 & 7.032 & 0,825 & 0,874 & 0,710 \\
\hline 68 Albania & 0,801 & 76,2 & 98,7 & 68,6 & 5.316 & 0,853 & 0,887 & 0,663 \\
\hline 69 Macedonia & 0,801 & 73,8 & 96,1 & 70,1 & 7.200 & 0,814 & 0,875 & 0,714 \\
\hline \multicolumn{9}{|l|}{ IDH medio } \\
\hline 84 Turquía & 0,775 & 71,4 & 87,4 & 68,7 & 8.407 & 0,773 & 0,812 & 0,740 \\
\hline 86 Jordania & 0,773 & 71,9 & 91,1 & 78,1 & 5.530 & 0,782 & 0,868 & 0,670 \\
\hline 88 Líbano & 0,772 & 71,5 & - & 84,6 & 5.584 & 0,775 & 0,871 & 0,671 \\
\hline 91 Túnez & 0,766 & 73,5 & 74,3 & 76,3 & 8.671 & 0,808 & 0,750 & 0,739 \\
\hline 104 Argelia & 0,733 & 71,7 & 69,9 & 73,7 & 7.062 & 0,778 & 0,711 & 0,711 \\
\hline 106 Palestina & 0,731 & 72,9 & 92,4 & 82,4 & - & 0,799 & 0,891 & 0,505 \\
\hline 108 Siria & 0,724 & 73,6 & 80,8 & 64,8 & 3.808 & 0,811 & 0,755 & 0,607 \\
\hline 112 Egipto & 0,708 & 70,7 & 71,4 & 76,9 & 4.337 & 0,761 & 0,732 & 0,629 \\
\hline 126Marruecos & 0,646 & 70,4 & 52,3 & 58,5 & 4.555 & 0,757 & 0,544 & 0,637 \\
\hline
\end{tabular}

Fuente: Elaboración propia a partir de los datos del Informe sobre Desarrollo Humano, 2007-2008. Naciones Unidas, 2008. 
ción. Es verdad que en la mayoría de los países del norte del Mediterráneo el ranking generado por el IDH se corresponde con el generado por el índice del PIB, sin embargo, en la mayoría de los países del sur y del este no ocurre lo mismo. Tal es el caso de Túnez, Turquía y Argelia que, a pesar de tener un PIB superior al de Bosnia, Albania, Líbano y Jordania, el valor de su IDH les sitúa por detrás de todos ellos al tener unos índices de educación o de esperanza de vida inferiores. La misma situación se produce en Líbano respecto de Albania y Jordania; en Marruecos respecto de Siria y Egipto; o en Egipto respecto de Siria. A Libia, por el contrario, su mayor PIB le ha colocado en mejor posición que a otros países con índices de salud y de educación superiores.

Por otro lado, el acceso a la educación, a la salud, o a los recursos económicos y, en definitiva, los logros del desarrollo, no están, ni siquiera en los países desarrollados, igualmente distribuidos entre hombres y mujeres. Dado que el IDH no capta esas diferencias, al no desagregar los datos según el género, el Índice de Desarrollo relativo al Género (IDG), aunque utiliza los mismos indicadores, sí capta las diferencias al tener en cuenta la distinta esperanza de vida de hombres y mujeres, el desigual acceso a la educación, o los distintos ingresos económicos que perciben. Los desequilibrios existentes entre los hombres y las mujeres en los países del Mediterráneo, en relación con las variables salud, educación e ingresos, se presentan en la Tabla 2. Se ha mantenido a la izquierda de cada uno de los países el número que les identifica con la posición del IDH para dejar constancia de que la posición en el ranking generado por el IDG no necesariamente coincide con la del IDH sino que, por el contrario, un país puede tener una posición mejor en el IDG si los logros del desarrollo son compartidos por igual entre hombres y mujeres. La mejor posición conseguida por los países del Mediterráneo en el ranking del IDG que en el del IDH, puede tener su explicación en el hecho de que Naciones Unidas aplica el IDG a menos países.

Una primera observación que puede hacerse, a partir de los datos de la Tabla 2, es que la única posición de ventaja de las mujeres, respecto de los hombres, tanto en los países del Mediterráneo como en los de todo el mundo, es la mayor esperanza de vida al nacer que las mujeres han conseguido. Ahora bien, esa diferencia de años de vida media no se da de manera uniforme en todo el Mediterráneo sino que guarda relación con el nivel de desarrollo de cada país: a un nivel de desarrollo mayor le corresponde una mayor diferencia en la esperanza de vida a favor de las mujeres y, por el contrario, a menor desarrollo la diferencia entre la esperanza de vida de los hombres y las mujeres se reduce. Así pues, si en la mayoría de los países del Mediterráneo con IDH alto la esperanza de vida de las mujeres es superior en seis o siete años a la de los hombres, en los países con IDH medio, la diferencia entre la esperanza de vida de los hombres y las mujeres es inferior a cuatro o cinco años. La mayor mortalidad de las mujeres relacionada con la maternidad (al no ser siempre atendidas por personal sanitario adecuado), el mayor número de embarazos y partos, el riesgo a contraer determinadas enfermedades o la precaria alimen- 
Tabla 2. Índice de Desarrollo relativo al Género

\begin{tabular}{|c|c|c|c|c|c|c|c|c|c|c|}
\hline \multirow[t]{2}{*}{$\begin{array}{l}\text { Clasificación } \\
\text { según el IDH }\end{array}$} & \multirow{2}{*}{\multicolumn{2}{|c|}{$\begin{array}{c}\text { Valor del } \\
\text { IDH }\end{array}$}} & \multicolumn{2}{|c|}{$\begin{array}{l}\text { Esperanza de } \\
\text { vida al nacer }\end{array}$} & \multicolumn{2}{|c|}{$\begin{array}{c}\text { Tasa de } \\
\text { alfabetización } \\
\text { (\% de } 15 \text { años y más) }\end{array}$} & \multicolumn{2}{|c|}{$\begin{array}{l}\text { Tasa bruta (\%) } \\
\text { combinada de } \\
\text { matriculación }\end{array}$} & \multicolumn{2}{|c|}{$\begin{array}{c}\text { Cálculo de los } \\
\text { ingresos percibidos } \\
\text { (PPA en \$ USA) }\end{array}$} \\
\hline & & & M & $\mathrm{H}$ & M & $\mathrm{H}$ & M & $\mathrm{H}$ & M & $\mathrm{H}$ \\
\hline \multicolumn{11}{|l|}{ IDH alto } \\
\hline 10 Francia & 7 & 0,950 & 83,7 & 76,6 & - & - & 99 & 94 & 23.945 & 37.169 \\
\hline 13 España & 12 & 0,944 & 83,8 & 77,2 & - & - & 101 & 95 & 18.335 & 36.324 \\
\hline 20 Italia & 17 & 0,936 & 83,2 & 77,2 & 98,0 & 98,8 & 93 & 88 & 18.501 & 39.163 \\
\hline 23 Israel & 21 & 0,927 & 82,3 & 78,1 & 97,7 & 97,7 & 92 & 87 & 20.497 & 31.345 \\
\hline 24 Grecia & 24 & 0,922 & 80,9 & 76,7 & 94,2 & 97,8 & 101 & 97 & 16.738 & 30.184 \\
\hline 27 Eslovenia & 25 & 0,914 & 81,1 & 73,6 & 96,6 & 99,7 & 99 & 90 & 17.022 & 27.779 \\
\hline 28 Chipre & 27 & 0,899 & 81,5 & 76,6 & 95,1 & 98,6 & 78 & 77 & 16.805 & 27.808 \\
\hline 29 Portugal & 28 & 0,895 & 80,9 & 74,5 & 92,0 & 95,8 & 93 & 87 & 15.294 & 25.881 \\
\hline 34 Malta & 33 & 0,873 & 81,1 & 76,8 & 89,2 & 86,4 & 81 & 81 & 12.834 & 25.623 \\
\hline 47 Croacia & 46 & 0,848 & 78,8 & 71,8 & 97,1 & 99,3 & 75 & 72 & 10.587 & 15.687 \\
\hline 56 Libia & 62 & 0,797 & 76,3 & 71,1 & 74,8 & 92,8 & 97 & 91 & 4.054 & 13.460 \\
\hline 66. Bosnia-H. & - & - & 77,1 & 71,8 & 94,4 & 99,0 & - & - & 2.864 & 4.341 \\
\hline 68 Albania & 61 & 0,797 & 79,5 & 73,1 & 98,3 & 99,2 & 68 & 69 & 3.728 & 6.930 \\
\hline 69 Macedonia & 64 & 0,795 & 76,3 & 71,4 & 94,1 & 98,2 & 71 & 69 & 4.676 & 9.734 \\
\hline \multicolumn{11}{|l|}{ IDH medio } \\
\hline 84 Turquía & 79 & 0,763 & 73,9 & 69,0 & 79,6 & 95,3 & 64 & 73 & 4.385 & 12.368 \\
\hline 86 Jordania & 80 & 0,760 & 73,8 & 70,3 & 87,0 & 95,2 & 79 & 77 & 2.566 & 8.270 \\
\hline 88 Líbano & 81 & 0,759 & 73,7 & 69,4 & 93,6 & 93,6 & 86 & 83 & 2.701 & 8.585 \\
\hline 91 Túnez & 83 & 0,750 & 75,6 & 71,5 & 65,3 & 83,4 & 79 & 74 & 3.748 & 12.924 \\
\hline 104 Argelia & 95 & 0,720 & 73,0 & 70,4 & 60,1 & 79,6 & 74 & 73 & 3.546 & 10.515 \\
\hline 106 Palestina & - & - & 74,4 & 71,3 & 88,0 & 96,7 & 84 & 81 & - & - \\
\hline 108 Siria & 96 & 0,710 & 75,5 & 71,8 & 73,6 & 87,8 & 63 & 67 & 1.907 & 5.684 \\
\hline 112 Egipto & - & - & 73,0 & 68,5 & 59,4 & 83,0 & - & - & 1.635 & 7.024 \\
\hline 126Marruecos & 112 & 0,621 & 72,7 & 68,3 & 39,6 & 65,7 & 55 & 62 & 1.846 & 7.297 \\
\hline
\end{tabular}

Fuente: Elaboración propia a partir de los datos del Informe sobre Desarrollo Humano, 2007-2008. Naciones Unidas, 2008. 
tación, son, entre otros, los responsables de que en los países menos desarrollados las diferencias en la esperanza de vida de hombres y mujeres sean menores.

Respecto a los niveles de educación, estos reflejan que, si bien la tasa de matriculación actual de las mujeres en muchos países es ligeramente superior a la de los hombres, sin embargo, la mayor tasa de alfabetización registrada por los hombres en todos los países revela la existencia de una mayor proporción de mujeres analfabetas en todos ellos, fundamentalmente en los países de IDH medio. Argelia, Egipto y Marruecos son los países con mayor proporción de mujeres mayores de 15 años analfabetas: concretamente, el $40 \%$ de las mujeres argelinas y egipcias mayores de 15 años y el $60 \%$ de las mujeres marroquíes son analfabetas.

En cuanto a la desigualdad de género atendiendo a los ingresos económicos percibidos, si bien es un hecho, además de injusto, universal, se pueden también apreciar importantes diferencias según el nivel de desarrollo y bienestar de los países. Es decir, en los países con IDH medio las diferencias entre los ingresos percibidos por las mujeres y por los hombres son más acentuadas que en los países con IDH alto.

Así pues, en ningún país con IDH alto los ingresos percibidos por los hombres llegan a ser el doble de los percibidos por las mujeres, aún siendo superiores en todos los casos. Los ingresos percibidos por las mujeres de los países del Mediterráneo con IDH alto son de media un 27\% inferiores a los de los hombres. Los países con menos desequilibrios entre los ingresos percibidos por los hombres y las mujeres son: Croacia, Portugal e Israel. Por el contrario, en los países de IDH medio los ingresos percibidos por los hombres son más del doble de los percibidos por las mujeres llegando en algunos países a ser tres o más veces superiores. Dicho en otros términos, los ingresos percibidos por las mujeres de los países del Mediterráneo con IDH medio son un $50 \%$ inferiores a los percibidos por los hombres. En países como Líbano, Jordania, Túnez, Argelia, Siria, Egipto y Marruecos, los ingresos percibidos por las mujeres son más del $50 \%$ inferiores a los que perciben los hombres. La situación más desigual se encuentra en Marruecos y Egipto (en ambos casos, los ingresos percibidos por las mujeres son un $60 \%$ inferiores a los de los hombres).

\section{El Mediterráneo: un mar de migraciones}

El Mediterráneo, más que una barrera, ha supuesto un vínculo entre distintos países que ha propiciado el desarrollo de los desplazamientos humanos hasta tal punto que no se podría entender la vida cultural, política, social y económica de esta región sin el intercambio de personas que se ha producido históricamente desde unas regiones a otras. Actualmente este intercambio, a diferencia de lo que ocurría en otros tiempos, aparece como manifestación de las desigualdades cada día más acentuadas entre países ricos y países pobres y que en el Mediterráneo encuentran un marco idóneo en el que mostrarse, como hemos visto en las páginas precedentes. 
Las migraciones en el Mediterráneo, tradicionalmente, se han abordado en cuatro ejes diferenciados: el Magreb, la Europa balcánica (repúblicas ex-yugoslavas, Albania, Turquía), Oriente Próximo y la Europa del Sur, perteneciente a la Unión Europea. En la actualidad, y dada la mundialización de las corrientes demográficas, se tratan como parte de un todo unitario; sin embargo, el desplazamiento de la población inmigrante sigue unas determinadas pautas.

La Europa comunitaria, especialmente Francia, ha sido y es el destino principal de los flujos migratorios procedentes del Magreb. Desde la descolonización de estos territorios, las metrópolis, especialmente Francia, se convirtieron en el destino de miles y millones de sus antiguos súbditos. Holanda, Bélgica, el norte de Italia y España también destacan por la gran presencia de inmigrantes magrebíes.

Los países de la Europa balcánica y del este, desde el derrumbe de sus modelos de estado y como resultado de la crisis económica y social que acompañó al cambio político, han proporcionado importantísimos contingentes de población a la Europa comunitaria. Alemania, Italia, Francia y España se han convertido en países receptores de rumanos, búlgaros, albaneses y eslovacos, entre otras nacionalidades de la región. Del mismo modo, Alemania ha sido el destino mayoritario de la inmigración turca, la cual constituye el principal colectivo entre los extranjeros residentes en el país teutón.

La industria derivada del petróleo ha sido el foco de atracción de distintos movimientos migratorios originados en Oriente Próximo y el Magreb. Arabia Saudita, Kuwait, los Emiratos Árabes Unidos (EUA), Bahrein, Omán y Qatar han acogido, desde los años ochenta, a más de seis millones de trabajadores extranjeros. Libia, gracias a la explotación de sus recursos naturales, también se ha convertido en receptor de inmigrantes originarios de sus países vecinos triplicando su población desde 1970. Esta zona de destino, en la ribera sur del Mediterráneo, también recibe importantes cantidades de población procedente del continente asiático, particularmente de Pakistán, Filipinas, Corea del Sur, Indonesia, Bangla Desh o Tailandia.

Todo ello corrobora y apoya que el término y concepto de mundialización de las migraciones esté aceptado en la actualidad por la comunidad científica y por los expertos en esta temática. En efecto, las migraciones no son un fenómeno numéricamente limitado: en 2004 en torno a un 2,5\% de la población mundial era emigrante. Los movimientos migratorios en el Mediterráneo son, en proporción, menos importantes en la actualidad que en tiempos de la diáspora hacia América o la colonización del continente africano. A pesar de que la amplitud de las migraciones sea limitada, sus efectos sobre las sociedades de acogida y llegada adquieren una vital relevancia. El impacto cultural y económico no es nada marginal, las transferencias financieras individuales - remesas- y su generalización constituyen una fuente de vida básica para ciertos países y se profundiza en unos nexos de unión que facilitan la transmisión de valores y pautas culturales desde los lugares de acogida. 
Aunque las redes migratorias, en muchas ocasiones, reproducen relaciones coloniales clásicas, como es el caso de los argelinos y marroquíes en Francia o de estos últimos también en España, el Mediterráneo ha sido y sigue siendo una zona de migración con fuertes disparidades, como se deriva de las cifras del IDH. Los países de la ribera sur y este poseen un porcentaje medio de población emigrante —el 5\%-, que dobla la tasa media mundial, siendo los principales países de salida: Turquía, Egipto, Marruecos y Argelia. Respecto al porcentaje de población inmigrante, como puede observarse en la Tabla 3 destacan, en la ribera este, países como Israel y Jordania (que, por otra parte, poseen un IDH mayor que el resto de los países del este y del sur), con una tasa próxima al 40\%, o Líbano con una tasa cercana al 20\%. Los países de la ribera norte con mayor proporción de población inmigrante son, en correspondencia con esos mismos datos (ver tabla 1), Francia, Croacia y España.

Por otra parte es importante destacar que los flujos migratorios en el Mediterráneo están íntimamente relacionados con los conflictos políticos, eco-

Tabla 3. Tasa de inmigración y nivel de IDH por países

\begin{tabular}{lccc}
\hline & Tasa de inmigración & Nivel de IDH & Población total \\
\hline Jordania & 38,6 & 0,773 & 5.759 .732 \\
Israel & 37,3 & 0,932 & 6.276 .883 \\
Líbano & 18,2 & 0,772 & 3.826 .018 \\
Libia & 10,9 & 0,818 & 5.759 .732 \\
Francia & 10,6 & 0,952 & 60.656 .178 \\
España & 9,9 & 0,949 & 45.200 .737 \\
Croacia & 9,6 & 0,850 & 4.495 .904 \\
Chipre & 6,3 & 0,903 & 780.133 \\
Serbia y Montenegro & 5,9 & & 10.829 .175 \\
Siria & 5,5 & 0,724 & 18.448 .752 \\
Grecia & 4,9 & 0,926 & 10.668 .354 \\
Italia & 4,8 & 0,941 & 58.103 .033 \\
Eslovenia & 2,6 & 0,917 & 2.011 .070 \\
Bosnia-Herzegovina & 2,4 & 0,803 & 4.025 .476 \\
Portugal & 2,3 & 0,897 & 10.566 .212 \\
Malta & 2,2 & 0,878 & 398.534 \\
Turquía & 2,2 & 0,775 & 69.660 .559 \\
Macedonia & 1,6 & 0,801 & 2.045 .262 \\
Argelia & 0,8 & 0,733 & 32.531 .853 \\
Albania & 0,4 & 0,801 & 3.563 .112 \\
Túnez & 0,4 & 0,766 & 10.074 .951 \\
Egipto & 0,2 & 0,708 & 77.505 .756 \\
Marruecos & 0,1 & 0,646 & 32.725 .847 \\
\hline
\end{tabular}

Fuente: elaboración propia elaboración propia a partir de los datos ofrecidos por las distintas oficinas estadísticas gubernamentales de los estados ribereños del Mediterráneo y por el Informe sobre el Desarrollo Humano 2007-2008 del Programa de Naciones Unidas para el Desarrollo (UNDP). 
Gráfico 1. Tasa de inmigración correlacionada con el nivel de IDH en los países mediterráneos.

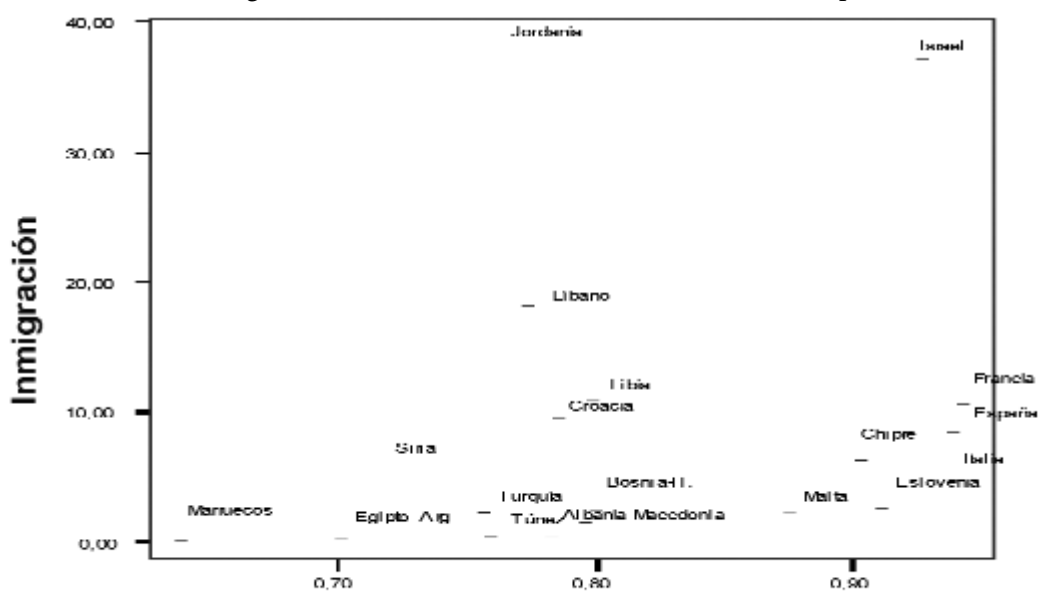

IDH

Fuente: elaboración propia a partir de los datos ofrecidos por las distintas oficinas estadísticas gubernamentales de los estados ribereños del Mediterráneo y por el Informe sobre el Desarrollo Humano 2007-2008 del Programa de Naciones Unidas para el Desarrollo (UNDP).

nómicos y culturales (Líbano, antigua Yugoslavia), pero que también dependen de la proximidad geográfica, como se observa en la diáspora de inmigrantes tunecinos o albanos a Italia.

Como consecuencia de la mundialización, las relaciones de la mayoría de los países con el resto del mundo se han acentuado. Variables como las exportaciones, la atracción de inversión extranjera directa, el porcentaje de la población con acceso a Internet, el registro de nuevas patentes, etc., son utilizadas por los países, para determinar si están teniendo éxito en este nuevo contexto. Todas estas son formas de participación en la mundialización desde arriba, sin embargo, la población pobre no es ajena a este proceso y, de hecho, también está participando de manera creciente en los procesos de mundialización, precisamente a través de las migraciones.

Según el Programa de Naciones Unidas para el Desarrollo (UNDP), las migraciones contribuyen al crecimiento general, a la mayor productividad, a un más alto nivel de empleo y a evitar el envejecimiento poblacional. Sin embargo, como se demuestra en el siguiente gráfico de correlación, una mayor tasa de inmigración no implica necesariamente un desarrollo humano más elevado. La inmigración depende, principalmente, de las oportunidades de empleo que ofrezca el país de destino, pero la existencia de éstas no garantiza que aspectos como la educación o la igualdad de género vayan siempre a la par.

Países como Jordania y Líbano se sitúan a la cabeza en cuanto a tasas de inmigración en el Mediterráneo y, sin embargo, sus niveles de IDH se sitúan 
por debajo de los de otros países en los que la inmigración es poco relevante, según queda reflejado en la Tabla 3 y en el Gráfico 1. En este último, y tras la utilización del coeficiente de correlación lineal de Pearson, se evidencia la no existencia de asociación lineal entre el índice de desarrollo humano (IDH) y la tasa de inmigración, ya que el estadístico resultante ofrece un valor muy próximo a cero. Sin embargo, resulta imprescindible señalar que, sin el efecto de países con un comportamiento atípico como Jordania y Líbano, con un IDH medio y una importante población inmigrante, sí que podríamos establecer correlación lineal entre ambas variables, puesto que el comportamiento típico de la mayoría de países es la correlación entre tasas de inmigración elevadas e índice de desarrollo humano también elevado, véase el caso de Francia, España, Italia o Israel y, al contrario, de Marruecos, Egipto, Túnez o Albania.

El que un país disponga de empleo para personas procedentes de fuera de sus fronteras no siempre significa que su sistema educativo, su sistema sanitario o sus mujeres se encuentren en una situación óptima, como se identifica en el Gráfico 1 en el que se correlaciona la tasa de inmigración con el índice de desarrollo humano de los países ribereños del Mediterráneo. Medir el desarrollo en términos humanos, considerando variables no únicamente económicas, facilita tener una visión integral más próxima a la realidad cotidiana de las personas.

\section{Las remesas mediterráneas, oportunidad para evitar los desequilibrios sociales}

De acuerdo con el World Economic and Social Survey 2005 sobre migración internacional, de Naciones Unidas, al inicio del siglo XXI más de 175 millones de personas, que representan casi el 3\% de la población mundial, residen fuera de su país de nacimiento. En el Magreb, por ejemplo, los emigrantes constituyen ya más del 15\% de la población. Estos emigrantes envían remesas al 17\% de los hogares de Argelia, Marruecos y Túnez. Las remesas que van desde España a Marruecos ascendieron en 2005 a 3.398 millones de dirhams (318 millones de euros). Esta cifra notable bastaría para sufragar el salario mínimo anual de 125.000 marroquíes.

Las remesas, del mismo modo, constituyen un importante alivio para la maltrecha economía argelina. La población en desempleo en este país se estima en torno a 1,5 millones de personas. La tasa de desempleo supera el 15\% según los datos arrojados en la último trimestre de 2007. Para los menores de 30 años este porcentaje se incrementa hasta el 75\%, cifra que equipara al conjunto de países de la ribera sur del Mediterráneo con muchos del continente americano.

El impacto económico de las migraciones no se limita a las remesas. Los migrantes, además de enviar remesas a sus familiares, realizan una diversidad de actividades culturales y socioeconómicas que los mantienen vinculados a su país de origen. Utilizan servicios de telecomunicaciones para relacionarse con sus familiares, contratan servicios de transferencias y envíos de dinero, deman- 
dan productos de consumo nostálgico y hacen inversiones en origen (compra de tierras, ganado, vivienda, pequeños negocios,...). Las remesas constituyen, por tanto, un reflejo de los fuertes lazos de reciprocidad y solidaridad que los inmigrantes mantienen, aún en la distancia, con sus comunidades y familias originarias. Son clara muestra de una intrincada combinación de dinámicas que interactúan en los niveles macroestructural, social y micro-individual, dentro de relaciones de transformación y dependencia que tienen la capacidad de promover e impulsar cambios de naturaleza social, cultural, económica y política.

Los ingresos que los emigrantes hacen llegar a sus lugares de origen son, según algunos expertos, un factor clave para la disminución de la pobreza. Para el año 2005, el Banco Mundial estimó que de los 232.000 millones de dólares USA pertenecientes al volumen de remesas mundiales en un ejercicio, 167.000 millones tuvieron como destino países en desarrollo, teniendo en cuenta que la cantidad es mayor si se contabilizan aquellas remesas que viajan por cauces informales de difícil control financiero.

En lo concerniente a las remesas y el desarrollo, el importe de las primeras es muy superior al de la Asistencia Oficial para el Desarrollo (AOD) y pronto alcanzará a las inversiones extranjeras directas (IED) como fuente de recursos. Para muchos países, como Marruecos, Argelia, Túnez o Egipto, las remesas son fuentes de ingresos estables, predecibles y ocupan una parte importante del Producto Interior Bruto (PIB). La remesa, como factor de desarrollo cultural y socioeconómico, evita la extrema pobreza de muchos hogares del sur del Mediterráneo incrementando el Producto Nacional Bruto (PNB) e, indirectamente, el Índice de Desarrollo Humano (IDH). Para el caso de Marruecos se estima que en el año 2003, más de un millón doscientos mil habitantes del país alahuita escaparon de la pobreza únicamente gracias al ingreso procedente de la solidaridad de sus familiares residentes en el extranjero, especialmente en países de la ribera norte mediterránea. Por ejemplo, el volumen de remesas recibidas en Marruecos se ha multiplicado por más de seis desde 1982 a 2003, pasando de 5.115.000€ a 34.733.000€, un crecimiento espectacular cuya tendencia sigue manteniéndose al alza con una ampliación paulatina del número de países emisores.

El riesgo de que con el tiempo se reduzca el flujo de las remesas y, con él, la posibilidad para muchos hogares de sobrepasar el umbral de la pobreza, se minimiza en la opinión de muchos autores, que cuestionan las consecuencias positivas de este maná a medio o largo plazo (Moré, 2005). Como es sabido, un importante volumen de las remesas se destina al consumo directo e inhibe la inversión productiva. Tanto en Marruecos como en Argelia, en las regiones en las que las remesas constituyen una parte mayor del ingreso familiar, se han abandonado cultivos tradicionales, se ha incrementado el desempleo e, incluso, se está asistiendo a la paradójica situación de admisión de mano de obra subsahariana para la ejecución de aquellas labores agrarias que las familias receptoras de remesas ya no desean llevar a cabo.

En el conjunto de la ribera sur del Mediterráneo quienes menos se benefician de las remesas son los más pobres, las familias de las regiones más desfa- 
vorecidas que, pese a lo que se pueda pensar, son quienes encuentran mayores dificultades para emigrar y, posteriormente, enviar dinero a sus lugares de origen. En este sentido, las remesas son otro factor de desigualdad que, si bien mitigan el hambre de hoy, propician la dependencia e inhiben cambios estructurales políticos y económicos necesarios en el sur del Mediterráneo para erradicar, de forma eficaz y definitiva, el hambre y la desigualdad.

Algunos autores y el Banco Mundial coinciden en que son necesarios mecanismos que faciliten el aprovechamiento de las remesas con el fin de promover a medio y largo plazo el crecimiento económico, reduciendo el coste de las transferencias y propiciando el ahorro de parte de las mismas para su aplicación en inversiones y en la obtención de créditos para la generación de actividades productivas.

Las remesas pueden convertirse en una oportunidad para el desarrollo, entendiendo este último no únicamente en su acepción económica. Para ello se hace imprescindible la ejecución de programas gubernamentales en los países receptores combinados con medidas supraestatales que encaucen las corrientes de remesas de los trabajadores hacia, por ejemplo, el desarrollo de infraestructuras y la creación de empleo que, como resultado, alienten a los trabajadores a no abandonar sus países y, de este modo, evitar la pérdida de capital humano, principal sangría socioeconómica de países como Marruecos, Argelia, Túnez, Egipto y Turquía. En estos últimos podemos encontrar alguna medida gubernamental de esta índole que, sin minusvalorar su enorme valor, no transciende el límite de lo anecdótico.

Además, las remesas poseen una dimensión estratégica que la ribera norte del Mediterráneo, especialmente la Unión Europea, puede aprovechar para reducir la presión migratoria que le afecta, presión motivada por la creciente desruralización del continente africano, especialmente del Magreb y de Turquía. Las transferencias personales de dinero procedentes de la emigración, junto con una activa política de cooperación al desarrollo, pueden ofrecer financiación adicional a las actividades productivas en áreas rurales.

Las migraciones constituyen un magnífico medio de comunicación cultural entre las dos riberas del mar mediterráneo. Si la mejora y disminución del precio del transporte ayuda a que no se produzca una ruptura definitiva con el pasado y a que los lazos étnicos y religiosos perduren y se refuercen en las dos orillas, es importante señalar que los nexos económicos se incrementan gracias a las remesas, el turismo y las inversiones. Si como indicaba Moré (2005), la frontera más desigual en términos económicos de la Unión Europea, o incluso de la Organización para la Cooperación y el Desarrollo Económico (OCDE) , es la que separa a las ciudades de Ceuta y Melilla con Marruecos, pues el Producto Interior Bruto (PIB) per cápita de España multiplica por quince al de Marruecos, en lo referente a la cultura, las fronteras son cada día más permeables por el efecto de las migraciones, de la presencia cada día más notable de los vecinos del sur en los países de la ribera norte.

En efecto, las redes sociales fruto de las migraciones, que vinculan el origen con el destino, conforman vías para comercializar bienes y servicios en el país 
de destino, a través de los canales convencionales o a través de negocios étnicos, que cubren una creciente demanda de mercado en los países con altas tasas de inmigrantes. Estas redes también sirven para la transmisión de información, conocimientos, ideas, actitudes, pautas de comportamiento, identidades y capital social. Es lo que se ha llamado remesas "sociales", de importante carácter cultural, que juegan un papel de gran relevancia en el desarrollo de los territorios como agentes de cambio, de transformación social y política.

\section{Conclusiones}

Las distintas situaciones demográficas por las que atraviesan los países de las riberas mediterráneas son las que en gran medida provocan actualmente y seguirán provocando en el futuro una presión migratoria del sur hacia el norte. Los diversos comportamientos ante la fecundidad y la mortalidad y sus diferentes crecimientos demográficos han abocado en unas estructuras de población jóvenes, en unos casos, y envejecidas, en otros, con necesidades muy dispares. Ese rápido crecimiento demográfico del sur del Mediterráneo es, entre otros aspectos, el causante de que las inversiones nacionales y extranjeras no tengan suficiente efecto sobre la eliminación de la pobreza. Todo ello justifica suficientemente el desarrollo del Proceso de Barcelona y de las estrategias de cooperación entre las distintas partes del Mediterráneo para conseguir el despegue de los países socios del sur y del este, el incremento de la inversión directa hacia ellos, y la formación y cualificación de sus poblaciones; en definitiva, la cooperación en el desarrollo de los socios mediterráneos del sur y del este para acabar así con las divisiones y desigualdades en la cuenca del Mediterráneo.

Por otro lado, las diferencias socioeconómicas en este contexto, lejos de reducirse, se están incrementando. El efecto de las remesas, si bien evita situaciones de máxima pobreza, no se ha convertido hasta el momento en un elemento clave del desarrollo de los países emisores de mano de obra. Sí están siendo, sin embargo, un elemento fundamental de transmisión cultural e ideológica y de creación de redes sociales. Del mismo modo, se corrobora que el desarrollo humano y las tasas de inmigración no correlacionan de forma evidente en una región mediterránea caracterizada, desde antaño, por las migraciones y por los desequilibrios sociales y demográficos.

Las migraciones hay que interpretarlas, no sólo como una fuente de desarrollo para los países emisores, sino también por su contribución a la sostenibilidad económicas de los países receptores. En definitiva, habría que hablar más de codesarrollo que de desarrollo, lo que supone entender las relaciones entre los países de las riberas del Mediterráneo de manera horizontal más que de forma vertical. 


\section{Bibliografía}

Algado, M. T. (2005). «La transición demográfica en el Mediterráneo». Sociedady Utopia. Revista de Ciencias Sociales.

Braudel, F. (1987). El Mediterráneo. Madrid: Espasa Calpe.

Castles, S. y Miller, M. J. (1998). The Age of Migration Internacional Population. Movements in the Modern World. Nueva York: The Guilford Press.

Collado, J. (2005). "Claves de los países mediterráneos en 2005». Economía exterior, n. ${ }^{\circ} 34$, España, Unión Europea, Mediterráneo.

Giménez Romero, C. (2003) ¿Qué es la inmigración? Barcelona: RBA.

Hammar, T., Brochmann, K. y FAIST, T. (2006) International Migration, Immobility and Development. Multidisciplinary Perspectives. Nueva York: Berg.

LEVITT, P. (1996) «Social Remittances: A Conceptual Tool for Understanding Migration and Development». Working Paper Series, n. ${ }^{0}$ 96.04. Center for Population and Development Studies, Harvard University.

López Bueno, J. M. (2005), «España-Marruecos: la frontera más desigual». Economía exterior, n. ${ }^{\circ}$ 34, España, Unión Europea, Mediterráneo.

Moré, I. (2005) Las remesas de los emigrantes en España: una oportunidad para la acción exterior. Documento de Trabajo (DT) 3/2005. Madrid: Real Instituto Elcano de Estudios Internacionales y Estratégicos.

Moreras, J. (1995) «Mar de migracions». En: DCIDOB, n. ${ }^{\circ}$ 51. Barcelona: Fundació Cidob.

Nyberg Sorensen, N. (2004) Migrant Remittances as a Development Tool. The Case of Morocco. Danish Institute for Development Studies.

SOLÉ, C. (2001) El impacto de la inmigración en la economía y en la sociedad receptora. Barcelona: Anthropos.

TAPINOS, G. (1996) «Development, Cooperation and Internacional Migration: The European Union and the Magreb». En: Mediterranean Conference on Population, Migration and Development. Palma de Mallorca, Estrasburgo: Consejo de Europa.

UNDP (Programa de Naciones Unidas para el Desarrollo) (2006). Estado de la Población Mundial. Nueva York: ONU.

UNDP (Programa de Naciones Unidas para el Desarrollo) (2006). Human Development Report. Beyond scarcity: Power, poverty and the global water crisis. Nueva York: ONU.

UNDP (Programa de Naciones Unidas para el Desarrollo) (2008). Informe sobre Desarrollo Humano. La lucha contra el cambio climático: solidaridad frente a un mundo dividido. Nueva York: ONU

UNDP (PROGRAMA DE NACIONES UNIDAS PARA EL DESARROLLO) (2005). World Survey on the Role of Women in Development: Women and International Migration. Nueva York: ONU.

VV.AA. (2005). Codesarrollo: migraciones y desarrollo. Madrid: CIDEAL.

Wihtol DE Wenden, C. "La inmigración en la cuenca mediterránea». Dossier La Vanguardia, n. ${ }^{\circ}$ 17. Barcelona: La Vanguardia. 